\title{
Technological Innovations in Emergency Department Services
}

\author{
(1) Perihan Șimșek¹, (1) Abdülkadir Gündüz², (1) Melih İmamoğlu³
}

\begin{abstract}
1Department of Emergency Aid and Disaster Medicine, Trabzon University, School of Applied Sciences, Trabzon, Turkey 2Department of Emergency Medicine, Karadeniz Technical University Faculty of Medicine, Trabzon, Turkey ${ }^{3}$ Clinic of Emergency Medicine, Rize State Hospital, Rize, Turkey
\end{abstract}

\begin{abstract}
Technology has brought various innovations on how healthcare providers acquire information, work, and communicate. These innovations are most common in the emergency department. Among the important innovations in the emergency department, imaging methods and diagnostic tests are now performed at the bedside. The main purpose of bedside imaging methods and diagnostic tests is to achieve rapid results and initiate appropriate treatment in a short time. Technological development provides fast access to diagnostic tests and rapid results as well as facilitates access to specialist physicians. Patients' data can be transmitted to advanced centers by telemedicine application, and consultation services can be obtained. Bedside consultation is also possible, thanks to the telemedicine application supported by robotic technology. Biosensors, mobile applications, technological products used in patient registration and follow-up process, and voice response systems used in patient monitoring after discharge are among other technological innovations used in emergency departments. All these tools and systems provide many facilities for emergency service delivery. However, reasons such as being expensive, not supervised by medical professionals, and time-consuming limit their use. While technological developments are increasing continuously, to ensure the same development in emergency departments, scientific studies with a high value of evidence are needed in this field.
\end{abstract}

Keywords: Digitalization, emergency department, innovation, technology

\section{Introduction}

Information and communication technology have advanced rapidly in the last two decades; Many new ways and tools have been developed in which information can be processed, stored and transmitted. The cloud-based data storage areas, wireless broadband networks, mobile computing systems and comprehensive data analysis methods affect the production and service industry fundamentally (1). The health sector is one of the areas that are influenced most by these rapid advances in information and communication technology. Advances in medical devices, equipment and diagnostic methods, as well as advances in information communication technology, bring important innovations to the functioning of the health system (2).

Emergency departments (EDs) have a special and important place in the health system. Scaling epidemics, disasters, accidents, and injuries in recent years increases the importance of EDs in the health system (3). On the other hand, the need for emergency EDs can reach a level that exceeds department resources. Technological advances provide important opportunities to meet the need for EDs by keeping service quality at the highest level. It is necessary to review ED services, redesign and strengthen them by making use of developing technological opportunities in order for the EDs to develop in parallel with the technological developments, and thus to provide the best service for the applications (2).

In this article, it is aimed to present information about technological innovations used in EDs. For this purpose, PubMed, Medline, Cochrane, and Google Scholar databases were searched using the keywords "emergency department", "digitalization", "technology" and "innovation". Literature research was expanded by examining the obtained publications and using the keywords "robotic technology", "mobile application", "wearable biosensor" 
and "artificial intelligence". Technological innovations used in EDs can be listed as; bedside imaging methods and diagnostic tests, decision support systems, artificial intelligence technology, telemedicine applications, robotic technology, wearable biosensors, mobile applications, technological innovations used in patient registration and follow-up, and voice response systems used in patient monitoring after discharge.

\section{Bedside Imaging Methods and Diagnostic Tests Bedside Ultrasonography}

The fact that ultrasonography (USG) can be applied at the bedside is an important technological development that enables fast diagnosis of life-threatening conditions. Bedside ultrasound brings convenience such as making or excluding key diagnoses, directing resuscitation noninvasively, reducing ionizing radiation, and ensuring safe implementation of invasive procedures (4). With the production of palm USG devices, these conveniences have been taken one step further. These devices, which are almost pocket sized, have the advantages of being both easy to use and cheap (5). In a systematic review, it was found that palm USG devices showed strong correlation with high-level ultrasound devices in the diagnosis of intra-abdominal free fluid and hydronephrosis, assessment of the pleural cavity, detection of abdominal aortic aneurysms, obstetric and gynecological examinations; however, it is stated that high evidence studies are needed to demonstrate its effectiveness in abdominal examinations (6). Despite its listed advantages and many conveniences, USG that can be applied at the bedside with today's technology cannot provide a complete radiological evaluation. Therefore, the need for conventional ultrasound devices continues when more extensive scanning is required (5).

\section{Portable Computer Tomography Devices}

Computed tomography (CT) is a powerful diagnostic method in detecting the presence of intracranial damage (7). However, during the transfer of patients who are sent to the imaging unit for brain CT procedure, problems such as failure to continue monitoring, interruption of intravenous therapy and inadequate ventilation may be encountered. These problems can cause many complications such as hypotension, hypoxia and increased intracranial pressure (8). Portable CT scanners, which have become widespread in recent years, have a capacity of taking about 32 images per second and can be brought directly to the patient's bed (9). This technology reduces the time required for imaging in the evaluation of patients suspected of stroke or head injury in EDs and can be effective in preventing complications that may arise during patient transfer (7).

With the developing technology, portable CT scanners have been produced that can display not only cranial imaging but also the whole body. Portable whole-body CT scanners, which can be readily carried from room to room, have proficiency to produce high standard CT imagery, and improve the diagnosis and treatment of a broad variety of emergency health problems. The whole-body CT scanners can give high-resolution bone, spine, thorax and soft tissue imaging of diagnostic value. However, abdominal images obtained by portable whole-body CT scanners are reported to be of lower quality than conventional CT imaging (7). Simultaneous display of multiple body areas with the portable whole body CT scanner both saves time and significantly reduces the dose of radiation exposure. In addition, the fact that these devices can be carried easily allows them to be taken to another unit and used for different clinical applications, when they are not needed in the $\operatorname{ED}(7,9)$.

\section{Bedside Tests}

Bedside tests are defined as any analytical test performed by a healthcare professional, outside the traditional laboratory setting for a patient. Terms such as near-patient testing, point of care testing, non-laboratory tests, decentralized laboratory tests are also used for bedside tests (10).

With the advances in microchip and miniaturization technology and developments in production processes in the last 10 years, fast, low cost, robust and reliable bedside measuring devices can be produced. With these devices, laboratory quality results can be obtained (11). It is also possible to transmit the results obtained from bedside testers wirelessly to the medical record system. Cartridges containing sensitive chemical biosensors are configured on the silicon chip to perform specific analyses for these devices. Thus, it is possible to carry out tests such as blood gas, biochemical, hematological, coagulation tests and $\beta$-HCG at the bedside. In addition, there are special tests to determine the basic metabolic status and serum lactate level, as well as special tests that can be used for the diagnosis of acute coronary syndrome and congestive heart failure $(10,12)$.

With bedside tests, necessary samples are taken for measurement in the patient's environment and test results can be obtained in a short time at the same place or near. Thus, the necessary treatment can be planned in a short time and near the patient (11). These tests allow rapid response to many emergency cases, such as immediate initiation of targeted therapy for patients with sepsis (13), cases of poisoning and overdose (14), and acute coronary syndrome (15).

\section{Decision Support Systems}

Tools that provide clinical decision support have a wide variety of computer-based systems, clinical guides, various algorithms, and digital resources that facilitate access to current information. 
Along with technological innovations, computer-based systems called advanced clinical decision support systems (CDSS) that can offer patient-specific recommendations have been developed (16). Advanced CDSS is an important innovation for EDs that require quick decision making in high-risk situations. These systems are used for various purposes in EDs (17).

With the development of clinical decision support systems, it was determined that better clinical management was provided in the EDs and better patient results were achieved. In the study of Niemi et al. (18), It has been shown that improvement has been achieved in the diagnosis and treatment of pneumonia and heart failure patients with the use of CDSS. Drescer et al. (19) determined that CDSS contributed significantly to the accuracy of the decision to have CT angiography in patients who were suspected of pulmonary embolism, but CDSS was considered to be less accepted by emergency physicians because it was timeconsuming. It has also been determined that with improved CDSS, there is a significant benefit in the management of sepsis cases (20), and in making the right triage decision (21).

Although clinical decision support systems have benefits and usefulness mentioned, they have some limitations and negative aspects. In a systematic analysis on this issue, the negative aspects are listed as follows. Clinical decision support systems can be installed as independent systems or integrated into the electronic health record (EHR) software of hospitals. In the case of the CDSS systems are a stand-alone system, it may disrupt the workflow of clinicians, as it requires clinicians to perform recording outside of routine computer work. If it is integrated into the system, it can create a substantial strain for the EHR system. In addition, clinical decision support systems can provide meaningless and unnecessary suggestions and warnings, and this can cause alert fatigue in healthcare professionals. Another limitation of decision support system is the difficulty in keeping the information bases of decision support systems up to date as clinical information changes. Moreover, the successful use of decision support systems requires proficiency in using advanced technology and these systems have a negative impact on user skill (16).

\section{Artificial Intelligence}

The usage area of artificial intelligence technology in health sector is getting wider. The first use of this technology in EDs is to provide decision support in triage (21). Today, artificial intelligence technology can be used in the EDs for patient monitoring and early detection of clinical complications (22).

Successful results are obtained in the evaluation of diagnostic imaging results such as computed tomography and USG with artificial intelligence technology in EDs (23). This technology has the potential to reduce the waiting time for radiology consultations in large centers and to enable rapid and accurate interpretation of imaging results in rural EDs where radiologists do not work (22).

Another usage area of artificial intelligence in EDs is natural language processing systems. These systems have the ability to interpret free texts, extract and classify certain words from these texts. Electronic clinical notes kept in the EDs can be analyzed with natural language processing systems and probability estimations can be made for various clinical situations (24).

\section{Telemedicine}

Telemedicine, which is defined as the remote provision of health services by using information and communication technology, enables healthcare professionals in hospitals with limited resources for acute developing serious health problems to connect to centers that are advanced in terms of expert and technical equipment and to receive consultancy services. Telemedicine applications in EDs are the best example of this (25).

Telemedicine is used to facilitate the management of different cases in EDs. In the EDs where emergency medicine specialists do not work, consultation can be provided with specialists for stroke cases by using telemedicine application (TeleStroke). Thus, the appropriateness of tissue plasminogen activator (tPA) treatment for cases can be determined and applied without delay (26). Telemedicine consultations were also found to contribute to accurate and rapid ECG assessment and initiation of appropriate therapy in cases followed up with suspicion of acute myocardial infarction (27).

Telemedicine technology is used in the management of psychiatric cases in EDs. With the service, also known as telepsychiatry, expert support on emergency psychiatric intervention can be provided (28). It has been found that management of sepsis cases with telemedicine is improved, especially in rural areas (29). Another area in which telemedicine technology is used is the maintenance of trauma cases care. In cases of trauma in rural areas, the frequency of mortality and morbidity is higher than in urban areas. With telemedicine, it is possible to consult the experts in developed centers about the cases of trauma in rural areas. Thus, access to definitive treatment can be shortened and early trauma case management can be improved (30).

\section{Robotic Technology}

In EDs, robotic technology can be used in the implementation of various tests that can assist in clinical risk classification. Concussion syndrome (CS) is a complication that can often be 
encountered after mild traumatic brain injury. Subbian et al. (31) developed a robot-supported objective neurological test that can guide clinical classification for CS and predict the probability of CS by evaluating neurological functions in EDs. However, in the study, it has been reported that difficulties such as creating a suitable area for the robotic device in the ED, requiring trained personnel to use the robot and providing special technical support for the maintenance of the device limit the use of the device in the EDs (31).

Another application area of robotic technology in EDs is telemedicine applications. While fixed monitors can be used for telemedicine, monitors can be taken to the patient by using mobile and remote-controlled robots. In the evaluation of stroke patients, O'Caroll et al. (32) found that the stroke team at the hospital was faster than the robotic telemedicine application. In the same study, it was reported that the results of robotic telemedicine applications were excellent and this method could be used safely for EDs that do not have a stroke team to intervene at the bedside.

\section{Mobile Applications}

Emergency physicians use many scoring systems and algorithms in their daily work. Mobile applications have been developed to provide easy access at the bedside to algorithms, checklists, clinical calculators and risk grading systems frequently used by physicians in EDs (33). Mobile applications used in EDs can be listed as providing decision support, accessing and evaluating patient data, and those that provide fast communication with other healthcare professionals or patients (34).

Mobile applications provide many conveniences to its users. Unlike other service tools used in EDs, it is abundant and much cheaper. However, those who develop these applications are not subject to quality standards and controls like other medical product developers. For this reason, mobile applications to be used must be carefully selected $(34,35)$.

\section{Wireless "Wearable" Biosensors}

Recent technological innovations show that wireless "wearable" biosensors can be used to catch the deterioration in the condition of patients at an early stage (36). In their study with sepsis patients, Garbern et al. (37) Determined that wireless "wearable" biosensors are a reliable way for continuous monitoring of heart rate and breathing. Another new use of wearable biosensors in EDs is to prevent deaths from overdose opioid intake, which is an increasing threat to the whole world. For this purpose, the drug dose used by patients who are prescribed opioids in EDs can be monitored with wearable biosensors. Thus, compliance of the patients with the treatment plan can be evaluated (38).

\section{Technological Innovations Used in Patient Registration and Follow-Up Online Registration}

In traditional EDs, every patient other than those coming to the ED by ambulance registers at the patient registry and the treatment priority is determined after the first assessment by the triage officer. With the developing technology, it is possible for the patients to make their own records for their ED visit from their homes and cars via their mobile phones. Thus, the workload of the employees in the triage and patient registration unit is reduced and it is possible to perform the admission processes in a shorter time (39).

Today, another innovation used for patient registration in EDs is kiosk devices. In addition to obtaining a queue number for examination, these patients can enter their medical history, background, pain level, and major complaints data into the system from the kiosk screen (40). Although a limited number of studies have been done on this new practice, the data obtained show that reduced workload helps triage nurses to focus more on patients' medical condition (41), and reduce the waiting time of patients in the $\mathrm{ED}(42)$.

\section{Patient Flow Software and Real-Time Location Systems}

The focus of ED service delivery shifts from expertise-based care to system-based key performance indicators such as "National Emergency Access Target (NEAT)" and "four-hour rule". This makes the patient transfer process and information transfer between emergency EDs and inpatient units more and more important (43).

Patient tracking systems and patient flow software are technology-based solutions developed to improve the transfer of patients from the emergency room to other service and diagnostic units in the hospital (44). Patient flow software used in the EDs enables patients' locations to be seen in the hospital. They can define the empty beds in the hospital according to their specialty. Thus, it contributes to the reduction of the waiting time of the patients who will be admitted from the ED, to facilitate the communication between the ED and other services, and to speed up the patient admission and discharge processes (45).

Patient tracking systems are based on infrared lights, radio waves and ultrasound technology. Patients are generally followed up with position transmission wristbands, and sensors and radios are used as tracking tools $(45,46)$. In some of these systems, by manually entering and updating data, patient information can be transferred and tracked from the ED to the inpatient units to which the patient is transferred. Some other systems provide tracking the location of patients in real time. Thus, it is possible 
to follow the units in which the patient in the ED is sent during the whole treatment process in real time (45).

With real-time follow-up, it is possible to monitor how long patients wait in the diagnosis and treatment units that they are sent to. In the system, it is also possible for healthcare professionals in the units where the patients go to share the information and notes they consider important to the patient. Along with the patient's location, important warnings such as "ready for discharge" or "waiting for the queue for X-ray" and "risk of falling" can be transferred to the system and monitored $(44,45)$. All this information can be seen on a digital screen called "electronic whiteboard" in the ED. Location transmission labels can also be applied to beds, staff and equipment, and allow tracking of all movements in the hospital (46).

Patient tracking systems and patient flow software provide ease of use of time more efficiently and increase safety on patient care (44). It is reported that the system facilitates the registration of the procedures performed on the patients, reduces the waiting time, and increases patient satisfaction (47). In addition, it provides hospital managers with important data to analyze the workflow. However, since patient tracking systems are very new, their effects and success outputs have not been fully documented (46).

\section{Voice Response Systems}

The follow-up of patients after discharge from the ED and the care requirements in the early discharge period are among the issues that draw attention in recent years. The most important problems related to patient safety in early discharge are not knowing what to do in case of symptoms restarting or progressing, and patients do not have their recommended controls (48). Depending on such problems, approximately $8 \%$ of the patients who are discharged from the ED may develop various complications after discharge (49). Telephone monitoring is carried out for the early detection of possible complications. This practice is reported to increase patient safety and satisfaction $(48,50)$. However, it is almost impossible to make phone calls with any patient who is discharged in the intensive working conditions of the EDs. Voice response systems have been used to overcome this limitation.

Interactive Voice Response System is an interactive system that enables the exchange of information by accessing information systems remotely by telephone, directing them with keys or voice. Thus, financial problems, personnel and time shortages can be overcome for the follow-up of patients discharged over the phone. The software owned by these systems also enables people to connect to a computer via the phone and transfer the data entered by the patients to the digital environment through the established connection $(49,50)$.

\section{Conclusion}

Technological innovations have provided significant benefits to the treatment and care process in EDs from patient admission to discharge. These benefits include reduced patient stay in the EDs, rapid diagnosis, real-time follow-up of patients in the EDs and hospital so that patient information can be shared with the entire care team, and medical errors due to causes such as memory factor and time pressure are avoided. The expeditious advances in technology have the potential to offer innovative solutions for predicting epidemics, avoiding preventable deaths, reducing healthcare waste, and improving efficiency and quality of care in ED services. However, in general, the use of technological developments in healthcare services brings along problems such as the safety and non-protection of patient data, weakening of caregiver-patient interaction, problems of integration and cost-effectiveness, and the inability of health professionals to adapt adequately to new technological changes. In conclusion, further studies with a high level of evidence regarding the effects of technological innovations on patient safety, care cost, and practitioner satisfaction are needed in order to overcome these problems and provide ED services with the highest quality using technological developments.

\section{Ethics}

Peer-review: Externally peer-reviewed.

\section{Authorship Contributions}

Concept: P.S., A.G., M.I., Design: P.S., A.G., Literature Search: P.S., Writing: P.S., A.G., M.I.

Conflict of Interest: No conflict of interest was declared by the authors.

Financial Disclosure: The authors declared that this study received no financial support.

\section{References}

1. Arugu LO, Chigozie CF. Information and communication technology (ICT) application in social and political system. European Journal of Research in Social Sciences. 2016;4:51-63.

2. Figueiredo J, Eiriz V. Analysis of the impact of technological innovation on healthcare services. International Journal of Behavioural and Healthcare Research. 2009;1:234-46.

3. Powell MP, Yu X, Isehunwa O, Chang CF. Trends in urgency of emergency department visits among those with and without multiple chronic conditions, 2007-2012. J Hosp Med Manage. 2016;2:1-9.

4. Chacko J, Brar G. Bedside ultrasonography: Applications in critical care: Part I. Indian J Crit Care Med. 2014;18:301-9.

5. Ali F, Ali E, Ali U, Hussain A. A narrative review on the advantages of portable ultrasound machines in the emergency department. Emergency Medicine and Critical Care. 2018;2:43-7.

6. Rykkje A, Carlsen JF, Nielsen MB. Hand-Held Ultrasound Devices Compared with High-End Ultrasound Systems: A Systematic Review. Diagnostics (Basel). 2019;9:61 
7. Rumboldt Z, Huda W, All JW. Review of portable CT with assessment of a dedicated head CT scanner. AJNR Am J Neuroradiol. 2009;30:1630-6.

8. Knight PH, Maheshwari N, Hussain J, Scholl M, Hughes M, Papadimos TJ, et al. Complications during intrahospital transport of critically ill patients: Focus on risk identification and prevention. Int J Crit IIn Inj Sci. 2015;5:25664.

9. Sempeles S. New Portable CT System Can Scan Entire Body. Journal of Clinical Engineering. 2011;36:86-7.

10. Patil S. POCTED: Use of point of care test devices in the emergency department. ICU Management \& Practice. 2016;16:154-6.

11. St John A, Price CP. Benefits of point-of-care testing in the Emergency Department, 2018. (cited 2019 April 9). Available at: https://acutecaretesting. org/-/media/acutecaretesting/files/pdf/benefits-of-pointofcare-testing-inthe-emergency-department

12. Hildyard C, Curry N. Point-of-care testing: a standard of care? Anaesthesia. 2015;70:1113-8.

13. Shim BS, Yoon YH, Kim JY, Cho YD, Park SJ, Lee ES, et al. Clinical Value of Whole Blood Procalcitonin Using Point of Care Testing, Quick Sequential Organ Failure Assessment Score, C-Reactive Protein and Lactate in Emergency Department Patients with Suspected Infection. J Clin Med. 2019;8:833.

14. Lager PS, Attema-de Jonge ME, Gorzeman MP, Kerkvliet LE, Franssen EJF. Clinical value of drugs of abuse point of care testing in an emergency department setting. Toxicol Rep. 2017;5:12-7.

15. Ahmed S, Jafri L, Raheem A, Shakeel S, Siddiqui I. Performance Evaluation of Cardiac Troponin I Assay: A Comparison Between the Point-of-care Testing Radiometer AQT90 FLEX and the Central Laboratory Siemens Advia Centaur Analyzer. Cureus. 2019;11:e4231.

16. Sutton RT, Pincock D, Baumgart DC, Sadowski DC, Fedorak RN, Kroeker KI. An overview of clinical decision support systems: benefits, risks, and strategies for success. NPJ Digit Med. 2020;3:17.

17. Bennett P, Hardiker NR. The use of computerized clinical decision support systems in emergency care: a substantive review of the literature. J Am Med Inform Assoc. 2017;24:655-8.

18. Niemi K, Geary S, Quinn B, Larrabee M, Brown K. Implementation and evaluation of electronic clinical decision support for compliance with pneumonia and heart failure quality indicators. Am J Health Syst Pharm. 2009;66:389-97.

19. Drescher FS, Chandrika S, Weir ID, Weintraub JT, Berman L, Lee R, et al. Effectiveness and acceptability of a computerized decision support system using modified Wells criteria for evaluation of suspected pulmonary embolism. Ann Emerg Med. 2011;57:613-21.

20. Bond CM, Djogovic D, Villa-Roel C, Bullard MJ, Meurer DP, Rowe BH. Pilot study comparing sepsis management with and without electronic clinical practice guidelines in an academic emergency department. J Emerg Med. 2013;44:698-708.

21. Fernandes M, Vieira SM, Leite F, Palos C, Finkelstein S, Sousa JMC. Clinical Decision Support Systems for Triage in the Emergency Department using Intelligent Systems: a Review. Artif Intel Med. 2020;102:101762.

22. Grant K, McParland A. Applications of artificial intelligence in emergency medicine. Univ Toronto Med J. 2019;96:37-9.

23. Jalal S, Parker W, Ferguson D, Nicolaou S. Exploring the Role of Artificial Intelligence in an Emergency and Trauma Radiology Department. Can Assoc Radiol J. 2021;72:167-74.

24. Ye Y, Tsui F, Wagner M, Espino JU, Li Q. Influenza detection from emergency department reports using natural language processing and Bayesian network classifiers. JAMIA. 2014;21:815-23.

25. Ward MM, Jaana M, Natafgi N. Systematic review of telemedicine applications in emergency rooms. Int J Med Inform. 2015;84:601-16.

26. Shea CM, Tabriz AA, Turner K, North S, Reiter KL. Telestroke Adoption Among Community Hospitals in North Carolina: A Cross-Sectional Study. J Stroke Cerebrovasc Dis. 2018;27:2411-7.
27. Miller AC, Ward MM, Ullrich F, Merchant KAS, Swanson MB, Mohr NM. Emergency Department Telemedicine Consults are Associated with Faster Time-to-Electrocardiogram and Time-to-Fibrinolysis for Myocardial Infarction Patients. Telemed J E Health. 2020;26:1440-8.

28. Freeman RE, Boggs KM, Zachrison KS, Freid RD, Sullivan AF, Espinola JA, et al. National Study of Telepsychiatry Use in U.S. Emergency Departments. Psychiatr Serv. 2020;71:540-6.

29. Mohr NM, Campbell KD, Swanson MB, Ullrich F, Merchant KA, Ward MM. Provider-to-provider telemedicine improves adherence to sepsis bundle care in community emergency departments. J Telemed Telecare. 2020:1357633X19896667.

30. Lapointe L, Lavallee-Bourget MH, Pichard-Jolicoeur A, Turgeon-Pelchat C, Fleet R. Impact of telemedicine on diagnosis, clinical management and outcomes in rural trauma patients: A rapid review. Can J Rural Med. 2020;25:31-40.

31. Subbian V, Ratcliff JJ, Meunier JM, Korfhagen JJ, Beyette FR Jr, Shaw GJ. Integration of New Technology for Research in the Emergency Department: Feasibility of Deploying a Robotic Assessment Tool for Mild Traumatic Brain Injury Evaluation. IEEE J Transl Eng Health Med. 2015;3:3200109.

32. O'Carroll CB, Hentz JG, Aguilar MI, Demaerschalk BM. Robotic telepresence versus standardly supervised stroke alert team assessments. Telemed J E Health. 2015;21:151-6.

33. Lin M, Rezaie S, Husain I. Top 10 mobile apps in emergency medicine. Emerg Med J. 2014;31:432-3.

34. Genes N. mHealth in emergency medicine [special report]. Emerg Med Pract. 2017;(Suppl 2017A):1-11

35. Yablowitz MG, Schwartz DG. A Review and Assessment Framework for MobileBased Emergency Intervention Apps. ACM Computing Surveys. 2018;51:1-32.

36. Breteler MJM MSC, Huizinga E, van Loon K, Leenen LPH, Dohmen DAJ, Kalkman CJ, et al. Reliability of wireless monitoring using a wearable patch sensor in high-risk surgical patients at a step-down unit in the Netherlands: a clinical validation study. BMJ Open. 2018;8:e20162.

37. Garbern SC, Mbanjumucyo G, Umuhoza C, Sharma VK, Mackey J, Tang O, et al. Validation of a wearable biosensor device for vital sign monitoring in septic emergency department patients in Rwanda. Digit Health 2019;5:2055207619879349.

38. Carreiro S, Wittbold K, Indic P, Fang H, Zhang J, Boyer EW. Wearable Biosensors to Detect Physiologic Change During Opioid Use. J Med Toxicol. 2016;12:255-62.

39. Thomas MC, Atrubin D, Hamilton JJ. Impact of Patient Self-Registration in Emergency Departments on Syndromic Surveillance Data. Online J Public Health Inform. 2015:7:e93.

40. Rhoads J, Drazen E. Touchscreen check-in: kiosks speed hospital registration. Oakland, CA: California HealthCare Foundation (online), 2009. (cited 2020 April 10): Available at: https://www.chcf.org/wp-content/uploads/2017/12/ PDF-TouchscreenCheckInKiosks.pdf

41. Coyle N, Kennedy A, Schull MJ, Kiss A, Hefferon D, Sinclair P, et al. The use of a self-check-in kiosk for early patient identification and queuing in the emergency department. Can J Emerg Med. 2019;21:789-92.

42. Mahmood A, Wyant DK, Kedia S, Ahn S, Powell MP, Jiang Y, et al. Self-CheckIn Kiosks Utilization and Their Association With Wait Times in Emergency Departments in the United States. J Emerg Med. 2020;58:829-40.

43. Staib A, Sullivan C, Cabilan CJ, Cattell R, Eley R. Digital transformation of the emergency department-inpatient interface (EDii): integration for future innovation. Aust Health Rev. 2020;44:666-71.

44. Pennathur PR, Cao D, Bisantz AM, Lin L, Fairbanks RJ, Wears RL, et al. Emergency department patient-tracking system evaluation. Int J Ind Ergon. 2011;41:360-9.

45. Gholamhosseini L, Sadoughi F, Safaei A. Hospital Real-Time Location System (A Practical Approach in Healthcare): A Narrative Review Article. Iran J Public Health. 2019;48:593-602 
46. Bacheldor B. Philly Hospital Uses RTLS to Track Patient Flow, Care and Training. RFID Journal. 2009;1-3.

47. Deryahanoglu 0, Kocaoglu B. Applications of RFID Systems in Healthcare Management: A Simulation for Emergency Department. IJITEE. 2019;10:78692.

48. Dhalla IA, O'Brien T, Ko F, Laupacis A. Toward safer transitions: how can we reduce post-discharge adverse events? Healthc Q. 2012;15:63-7.
49. Calder LA, Cwinn AA, Gatien M, Gee A, Larocque N, Calder-Sprackman S, et al. The feasibility of an interactive voice response system (IVRS) for monitoring patient safety after discharge from the ED. Emerg Med J. 2018;35:180-5.

50. Chern CH, How CK, Wang LM, Lee CH, Graff L. Decreasing clinically significant adverse events using feedback to emergency physicians of telephone followup outcomes. Ann Emerg Med. 2005;45:15-23. 